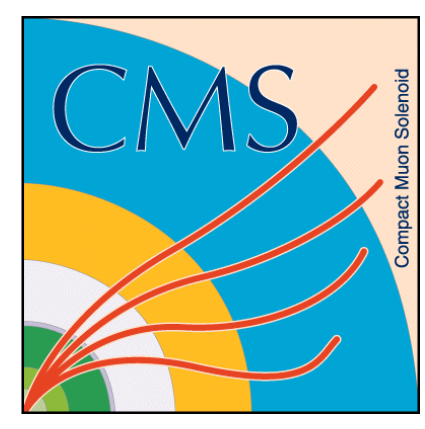

\title{
CMS HCAL Test Beam Results and Comparison with GEANT 4 Simulation
}

\author{
Jordan Damgov \\ on behalf of the \\ CMS HCAL Collaboration
}

CALOR '06 Chicago June 5-9, 2006 


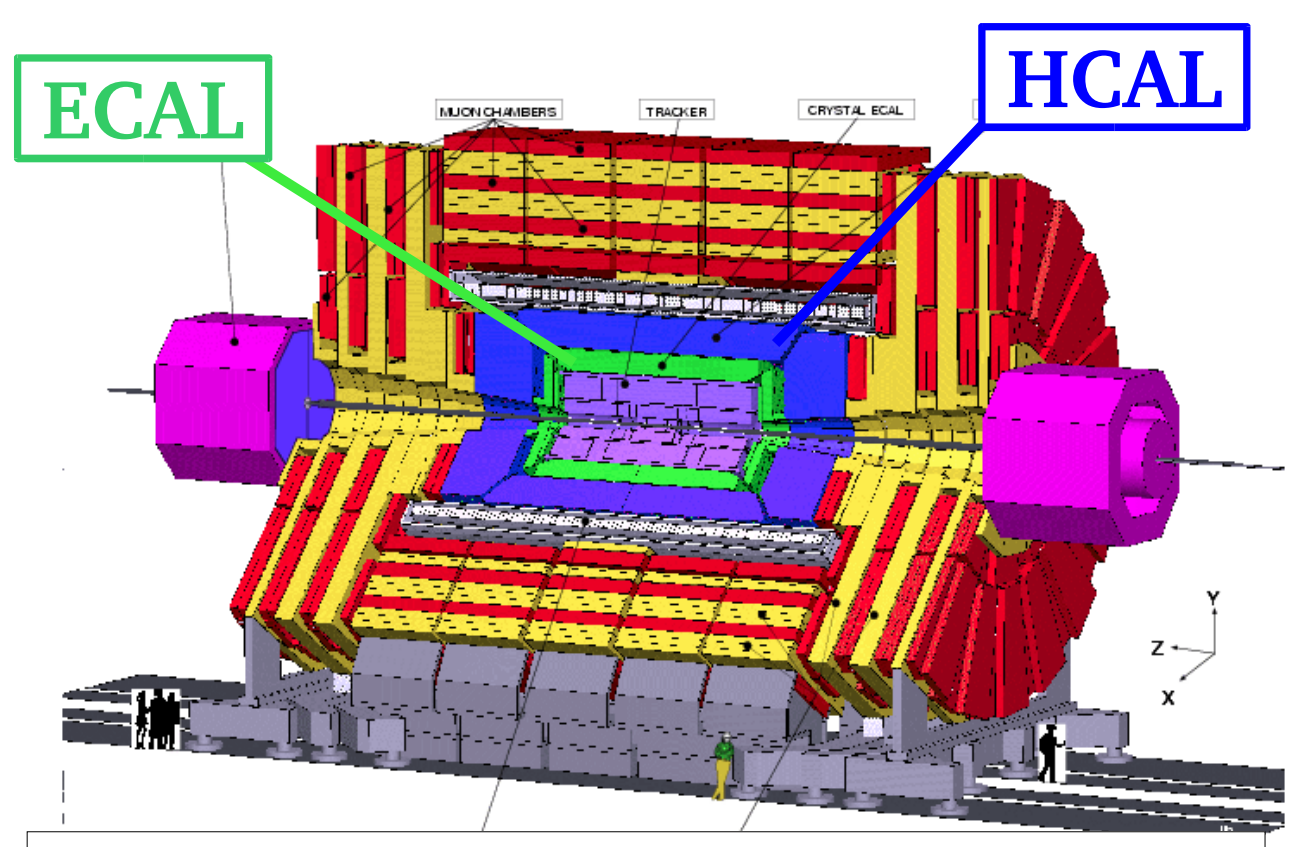

See Julie Whitmore's talk for details

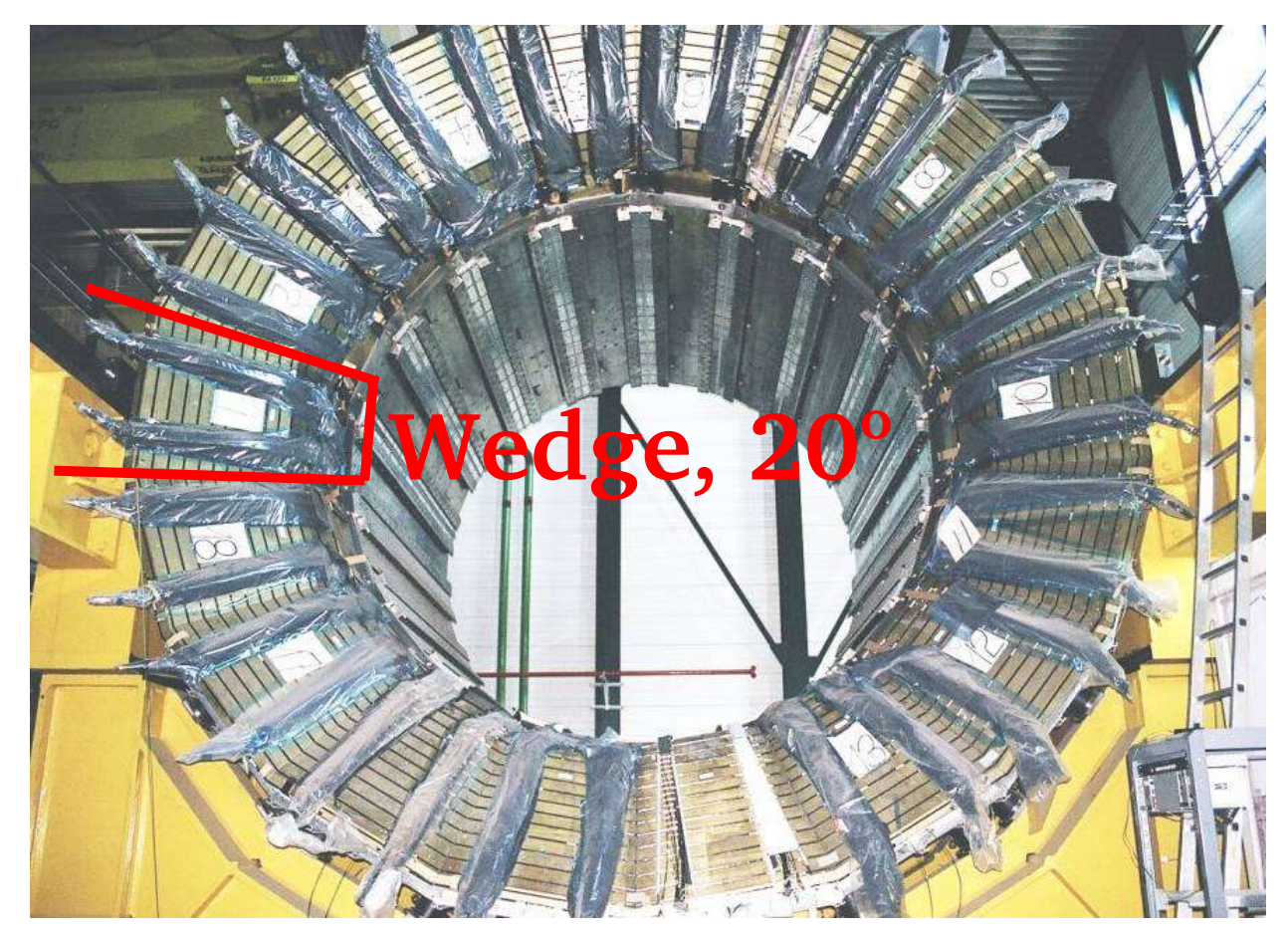

CMS Calorimeter system

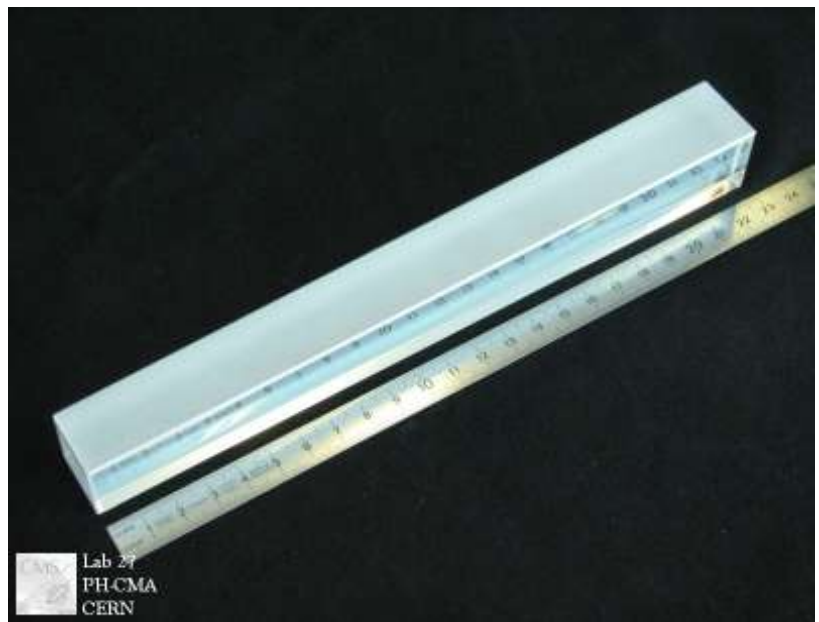

ECAL:

$\mathrm{PbWO}_{4}$

crystals

Sampling calorimeter Scintillator

Brass (70\%Cu,30\%Zn)

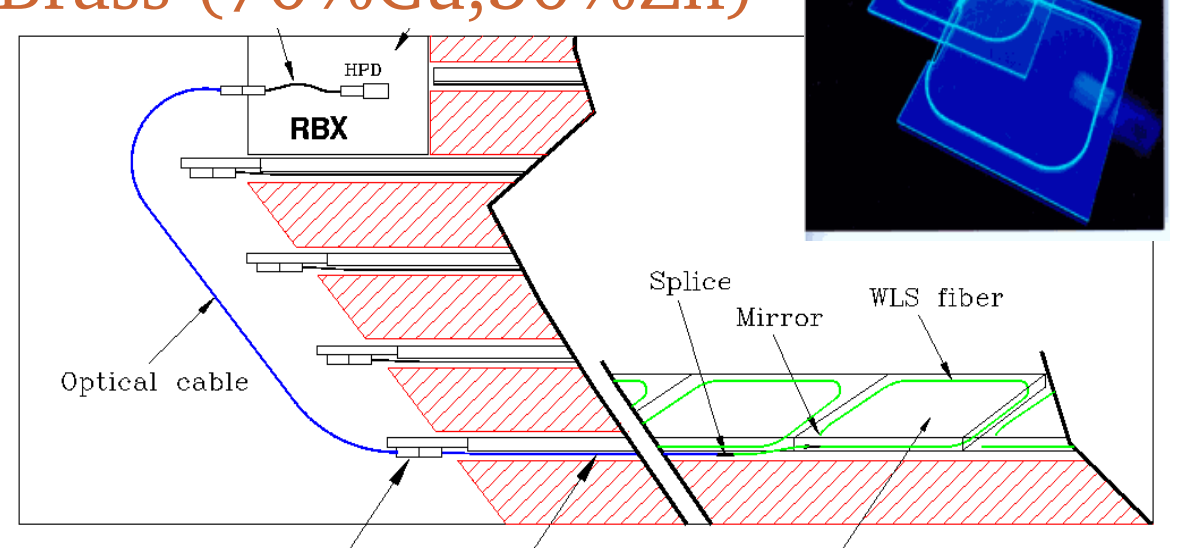

optical connector 17 Layers

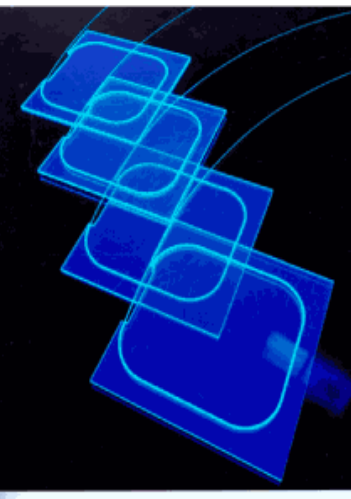

WLS fiber 


\section{Interaction point like geometry}

The test beam setup is designed to preserve the interaction point-like geometry of CMS.

Beam

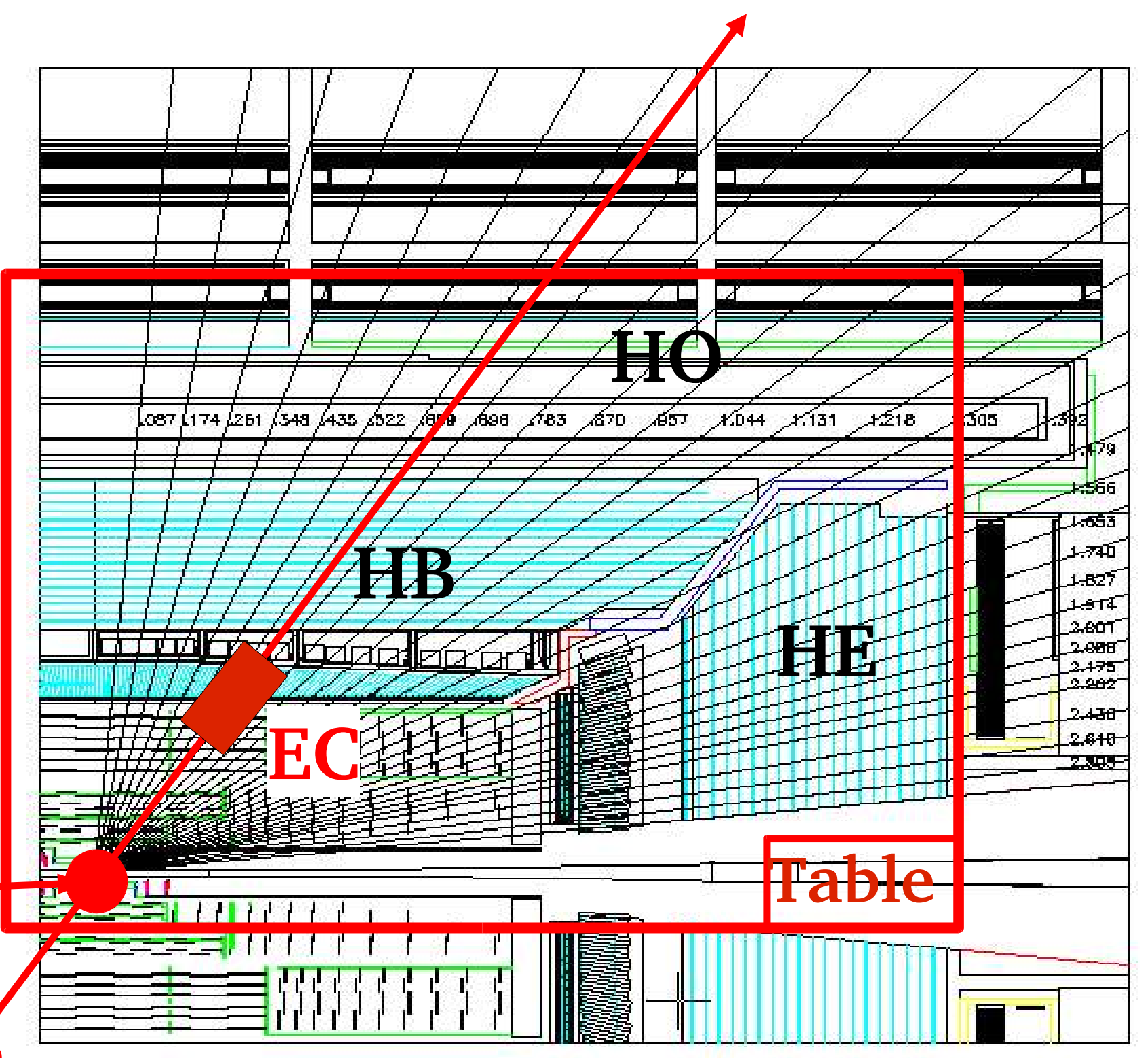




\section{Test beam setup}

2 Hadron barrel wedges 1 Hadron endcap wedge Hadron outer calorimeter 7x7 crystals ECAL Material for Magnet Movable table in $\eta-\phi$ plane

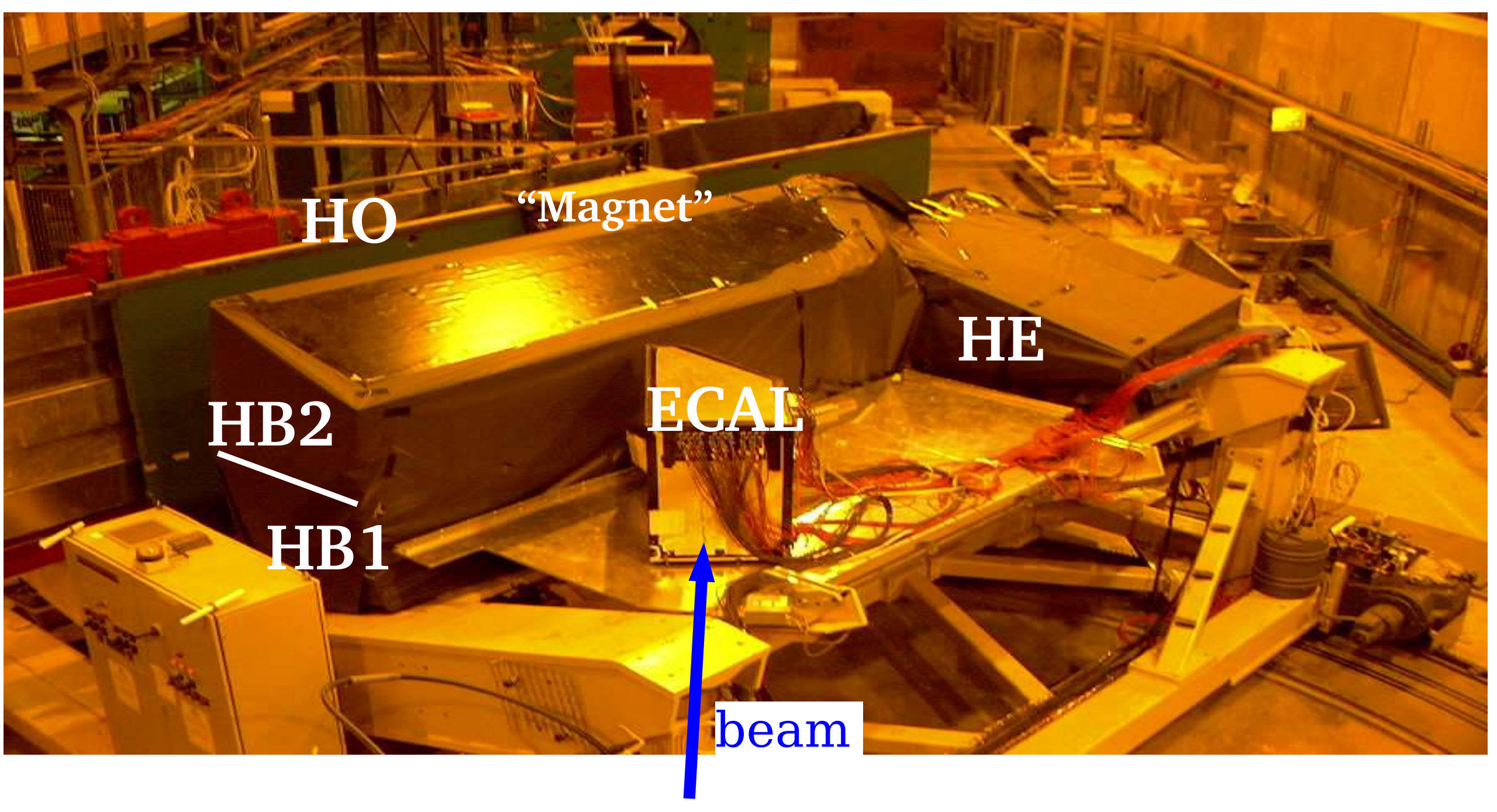




\section{ECAL and HO}

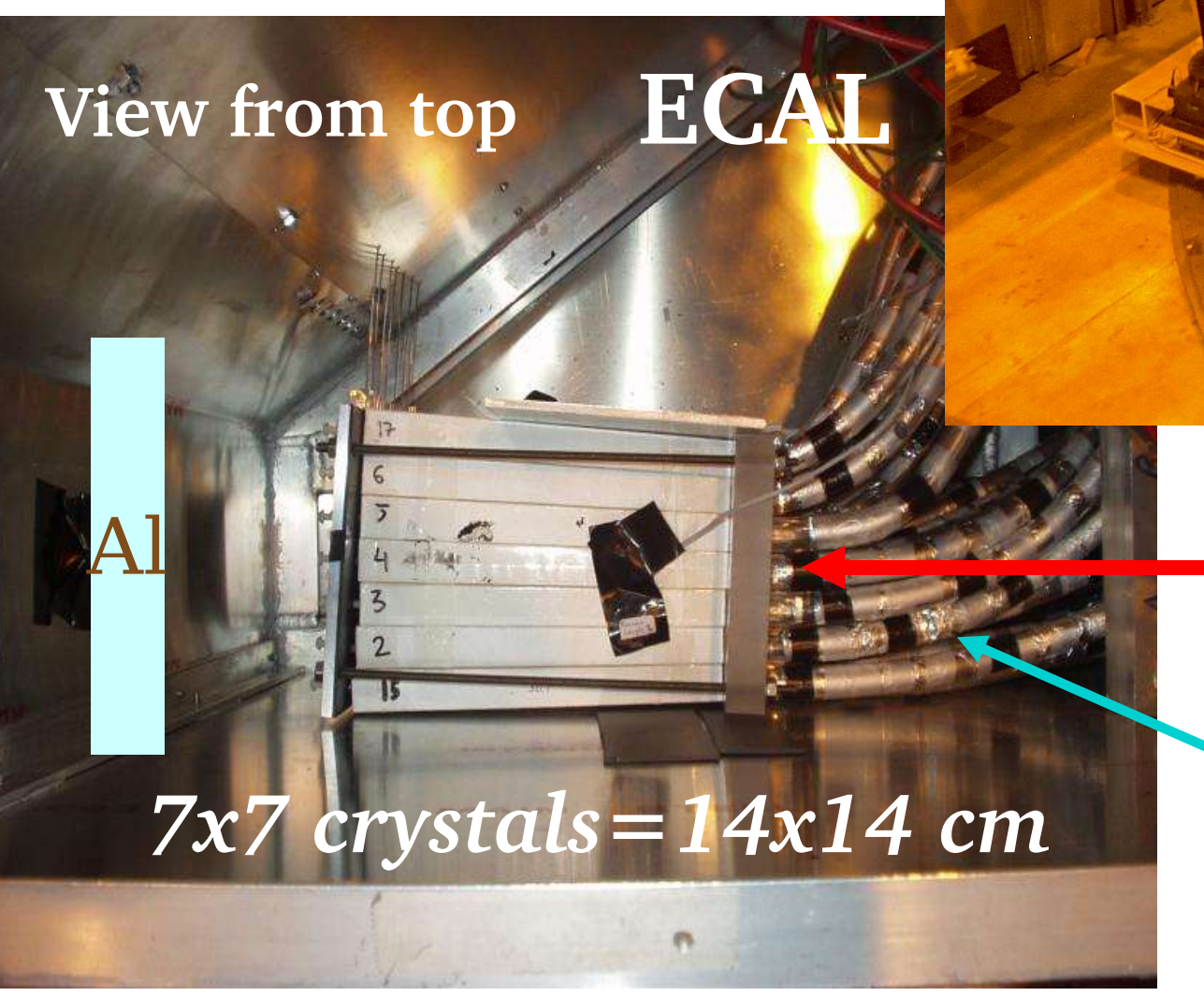

BEAM ECAL is readout by PMTs.

Light guides to PIMTs Light guides are attached to the front face of the crystals. 


\section{GEANT4 simulation}

-Detailed HCAL geometry with HB1\&HB2 read-out schema.

-ECAL - $\mathrm{PbWO}_{4}$ crystals, $\mathrm{Al}$ box and Al block behind ECAL. -Beam line - trigger counters and wire chambers

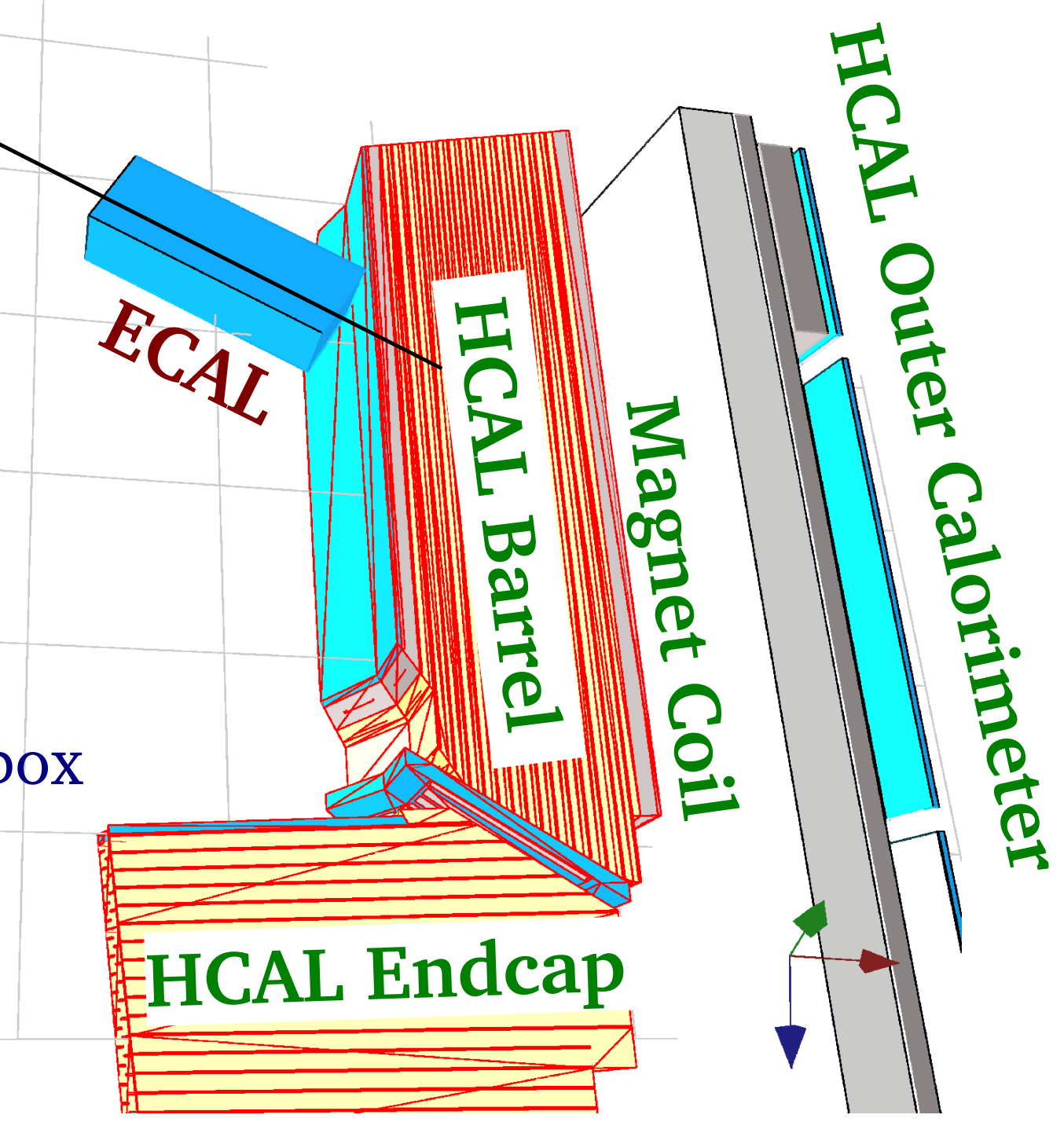




\section{GEANT4 physics models validation}

Physics lists tested against the test beam data :

-LHEP: LEP/HEP parametrized models for inelastic scattering.

-QGSP: Quark Gluon String model for the "Punch-through" interactions.

-QGSC: QGSP + Chiral invariant phase-space decay.

-FTFP: diffractive string excitation similar to that in FRITOF and Lund

In this comparison is used Geant 4.6.2.p02

Parametrized: LHEP-3.7

Model based: QGSP-2.8

QGSC-2.9 and FTFP-2.8 produce very similar to QGSP-2.8 results. 


\section{Beam line with particle identification 2-300 GeV/c}

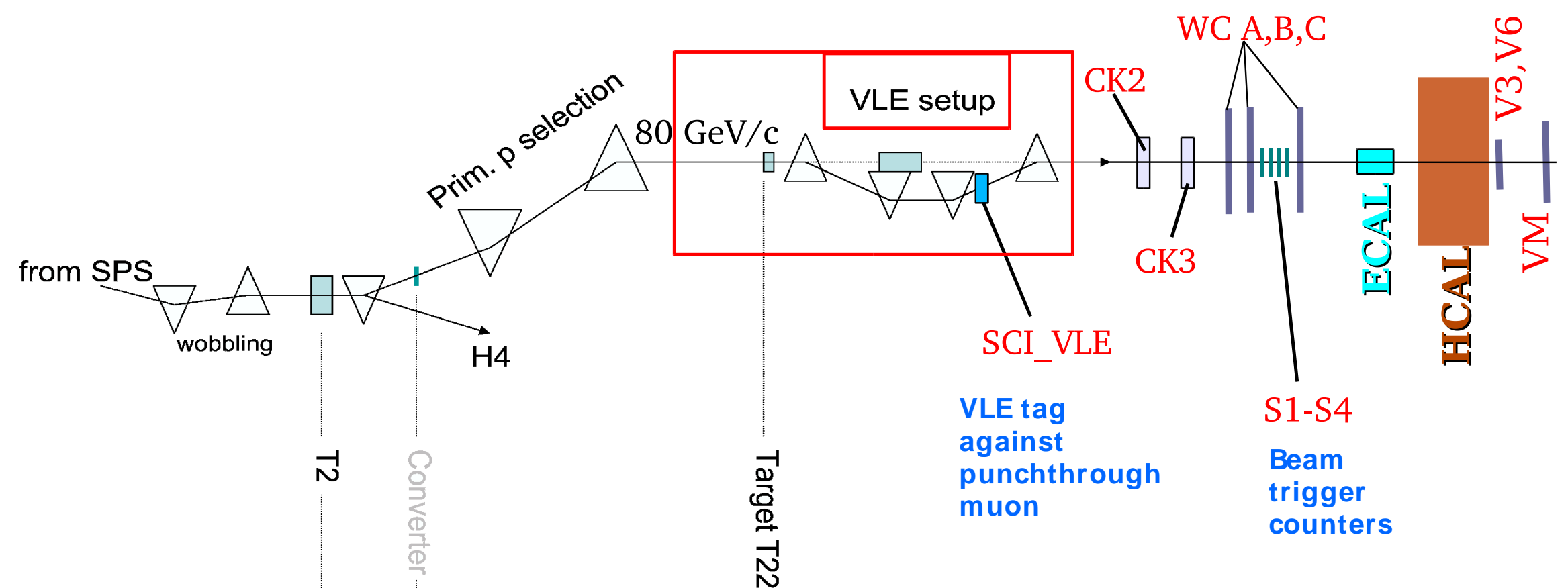

$400 \mathrm{GeV} / \mathrm{C}$

primary protons
$10 \mathrm{GeV} / \mathrm{c}<\mathrm{p}<400 \mathrm{GeV} / \mathrm{c}$

secondary hadrons/electrons
2-9 GeV/c tertiary

hadrons/electrons
Wire Chambers (WC A,B,C): single hit to reject

interaction in beam line

Available beam tunes:

pions 2-300 GeV

muons $80 / 150 \mathrm{GeV}$

electrons $9-100 \mathrm{GeV}$

\section{P-ID:}

Cerenkov counter (CK2) - electron

Cerenkov counter (CK3) - pion / kaon / proton Scintillators (V3, V6, VM) - muon tagging 


\section{Beam contamination and cleaning}

Beam contamination before the clean up:

\begin{tabular}{|ccr|}
\hline $\begin{array}{c}P_{\text {beam }} \\
{[\mathrm{GeV}]}\end{array}$ & $\begin{array}{c}\mathrm{mu} \\
{[\%]}\end{array}$ & $\begin{array}{r}\mathrm{el}- \\
{[\%]}\end{array}$ \\
\hline 300 & 0.7 & 0 \\
150 & 2 & 0 \\
100 & 2 & 0 \\
50 & 2.5 & 0 \\
30 & 3.5 & 7 \\
15 & 11 & 35 \\
10 & 7 & 70 \\
\hline 9 & 1.5 & 6 \\
7 & 3.5 & 10 \\
5 & 5.5 & 6 \\
\hline 3 & 30 & 30 \\
2 & 85 & 7 \\
\hline
\end{tabular}

+ interaction in the beam line

2 and $3 \mathrm{GeV}$ are not used
Beam cleaning:

- The particle Id counters are used for beam with momentum from 2 to $15 \mathrm{GeV}$

- Calorimeter based cuts: use the particle Id capabilities of the calorimeters.

High energy muons a tagged by the muon veto counters with 99\% efficiency. Low energy muons form pion decay are evaluated to be less then 1.5\% for $9 \mathrm{GeV}$ and below.

Electrons are clearly identify by ECAL/HCAL energy deposition. Any remaining uncertainty in the beam contamination and interaction in the beam line is added to the systematics of the measurement. 


\section{Uniformity calibration and Energy Scale}

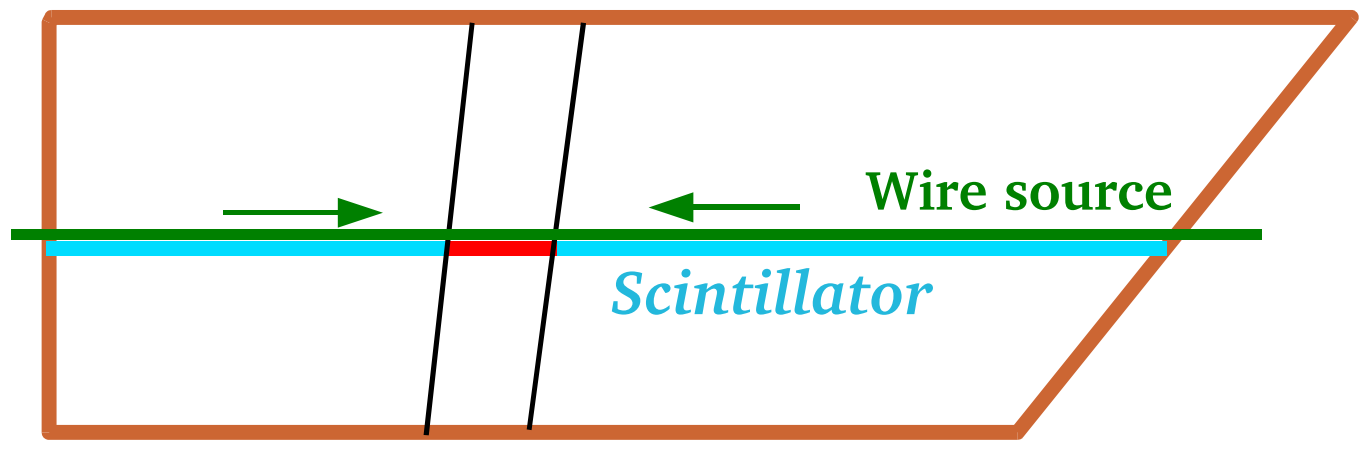

See Mayda Velasco's talk

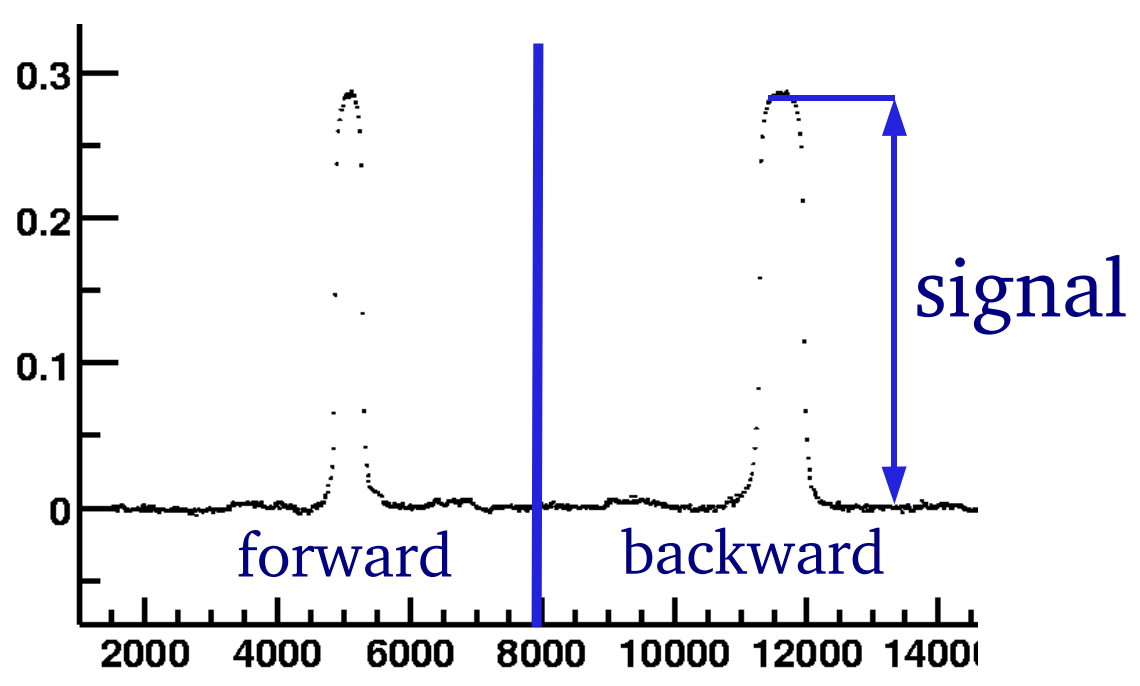

The uniformity calibration is done with $\mathrm{Co}^{60}$, per-tower and per-layer

\section{Reconstructed energy:}

$\mathrm{E}_{\mathrm{rec}}=\mathrm{a} * \mathrm{E}_{\mathrm{ECAL}}+\mathrm{b} * \mathrm{E}_{\mathrm{HCAL}}$

\section{Energy scale: ECAL: $100 \mathrm{GeV}$ e- HCAL: $50 \mathrm{GeV}$ pi- with MIP in ECAL.}




\section{Energy spectra ECAL+HCAL: data vs GEANT4}
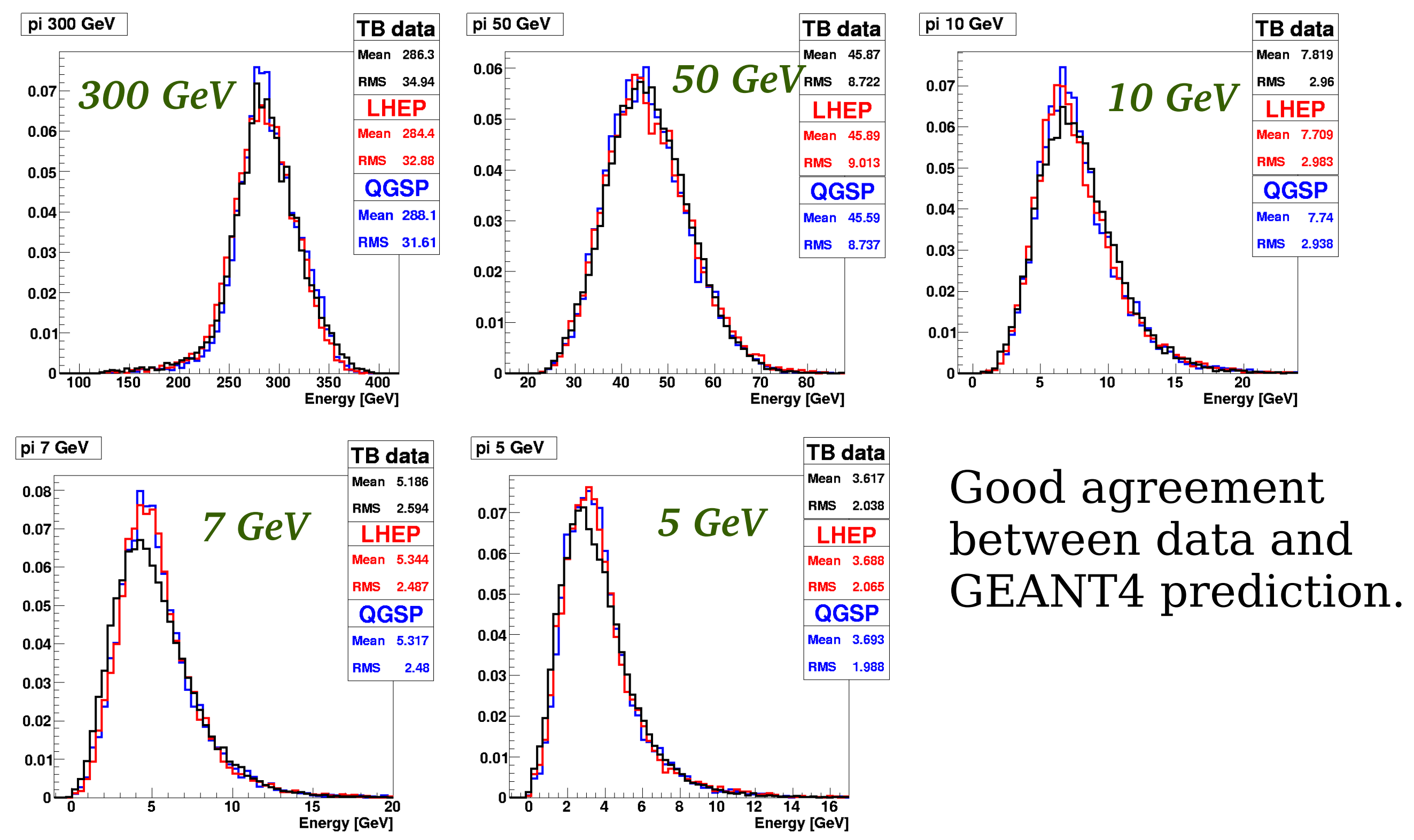


\section{Calorimeter response to pions}

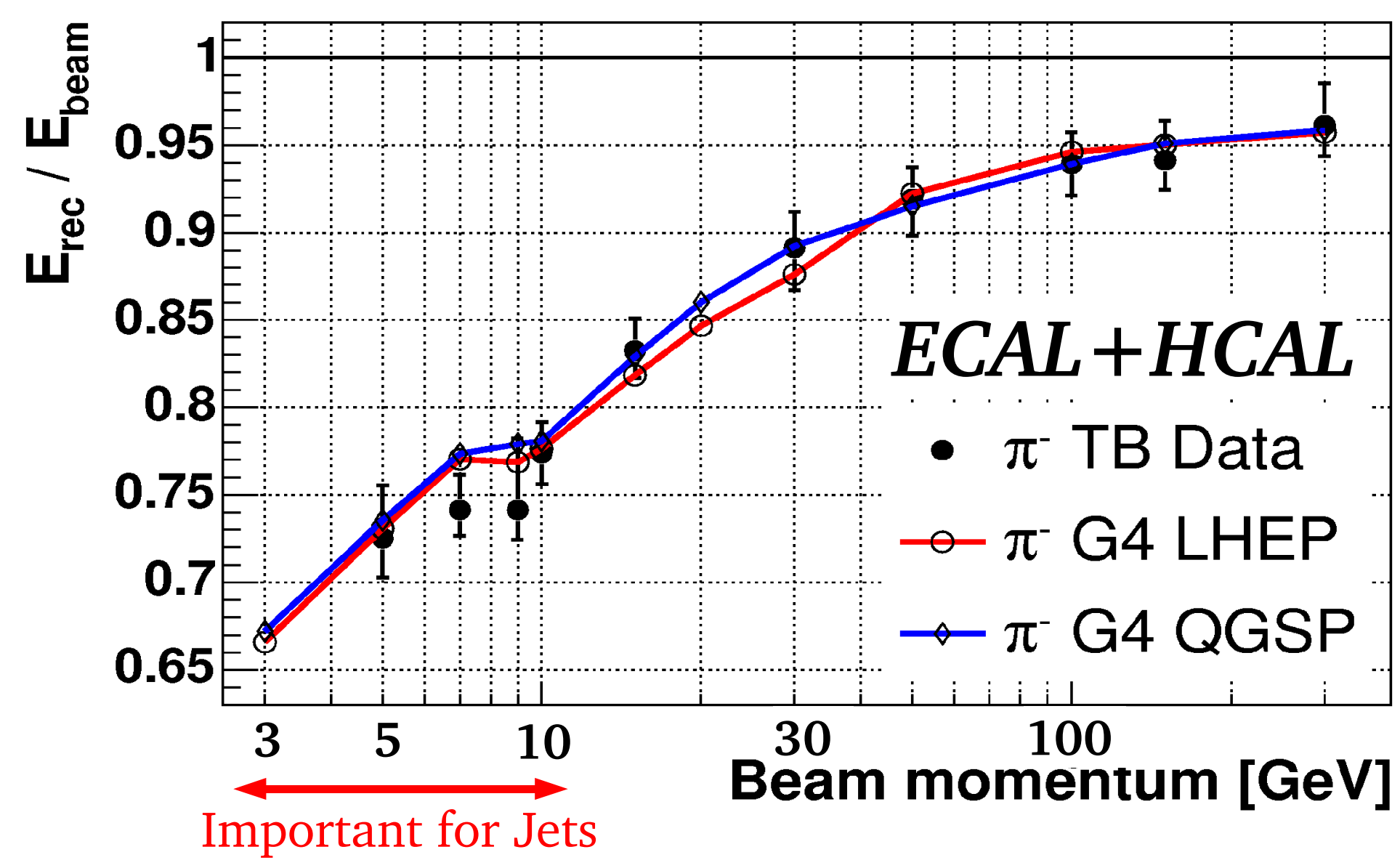

GEANT4 models correctly the calorimeter response to pions in broad energy range. Correct representation of the single hadron response at low energy is important for simulation of the calorimeter response to jets.

Some discontinuity is observed at $7-10 \mathrm{GeV}$ in the GEANT4 prediction. 


\section{HCAL alone response to pions}

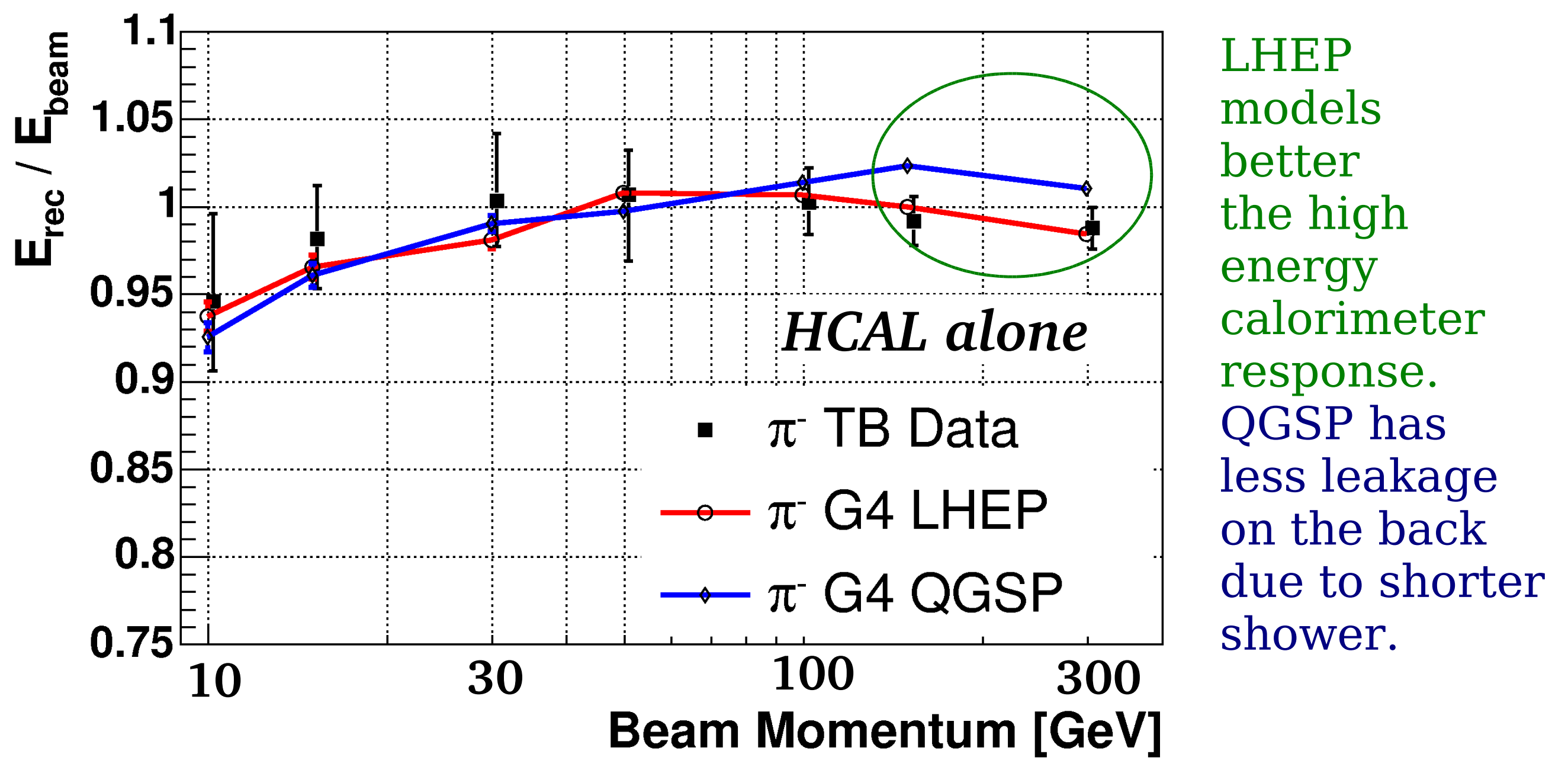

HCAL alone: MIP in ECAL is required. HO is not used in this measurement to compensate the HB leakage on the back 


\section{Proton over pion response ratio}

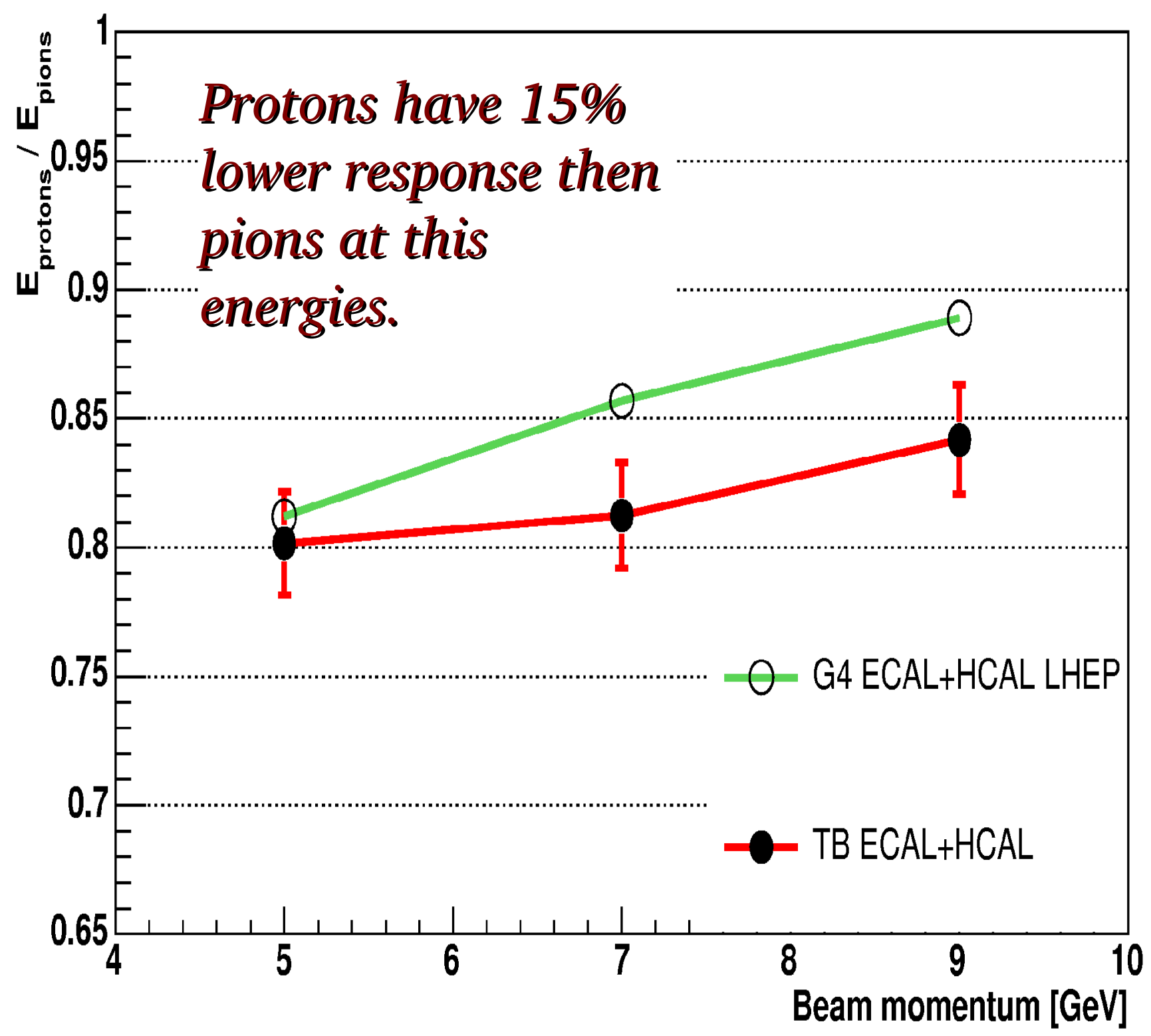

Significant difference in the calorimeter response to protons with respect to pions is observed in the data and is well represented in the GEANT4 simulation.

We will remeasure it this summer with improved particle Id. 


\section{Longitudinal shower profile measurement}

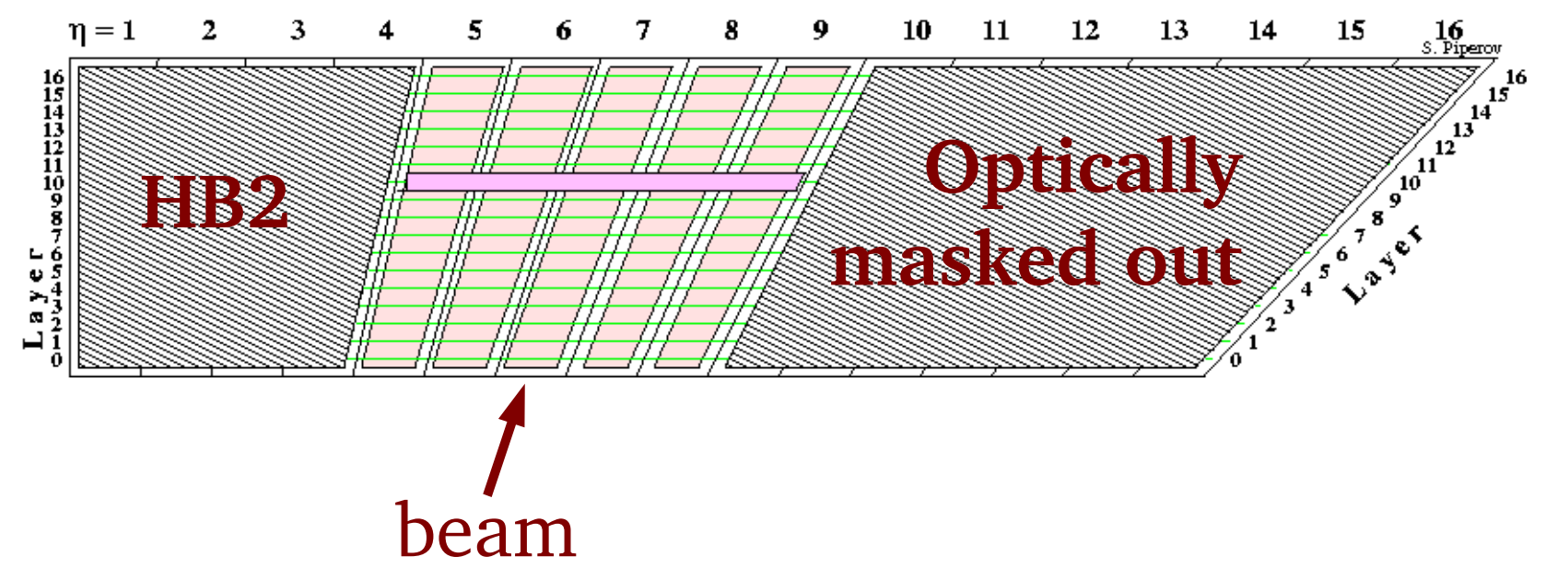

Modified read-out: redesigned optical decoding units to allow longitudinal shower measurement

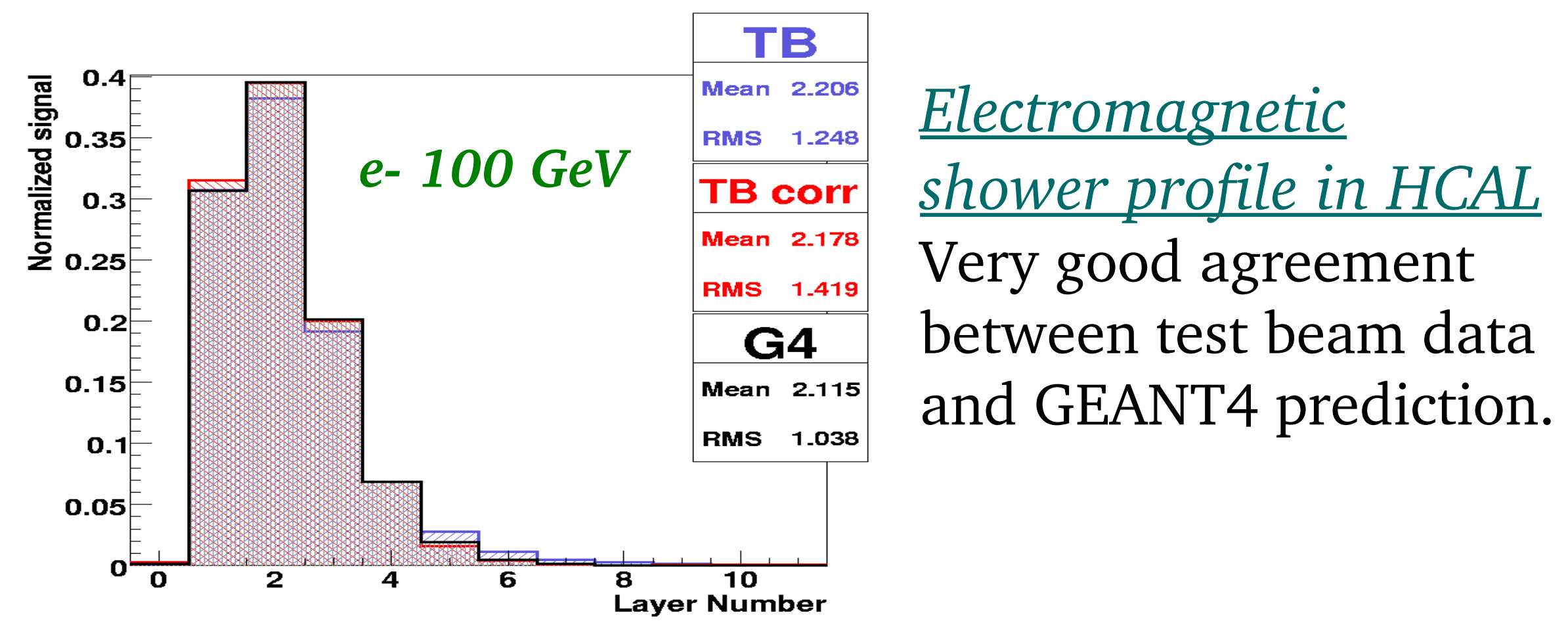




\section{Longitudinal shower profile measurement (cont.)}
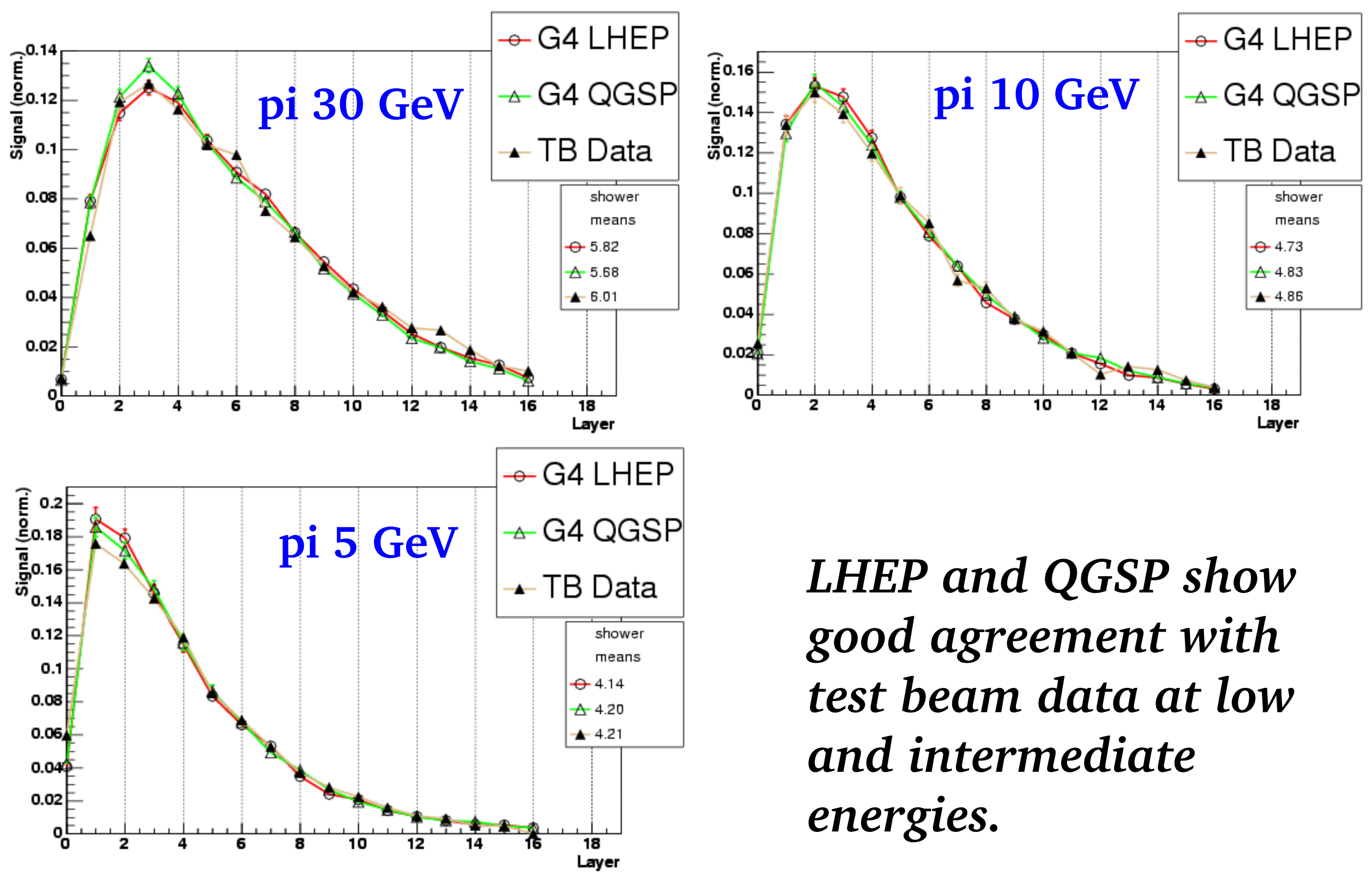


\section{Longitudinal shower profile measurement (cont.)}

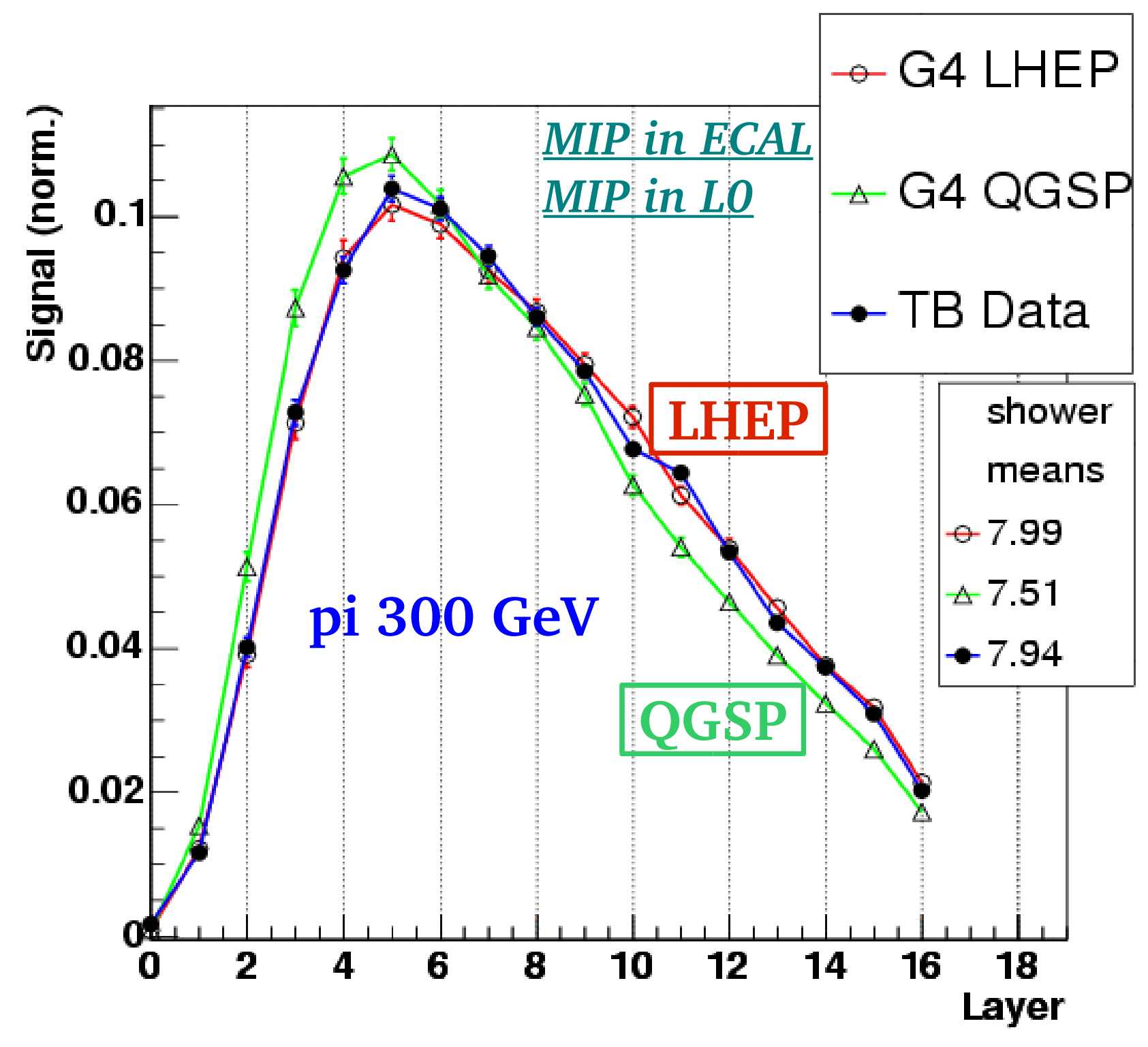

QGSP physics list has shorter shower profile for incident particles with high momentum. 


\section{HCAL - ECAL response to very low energy pion beam}
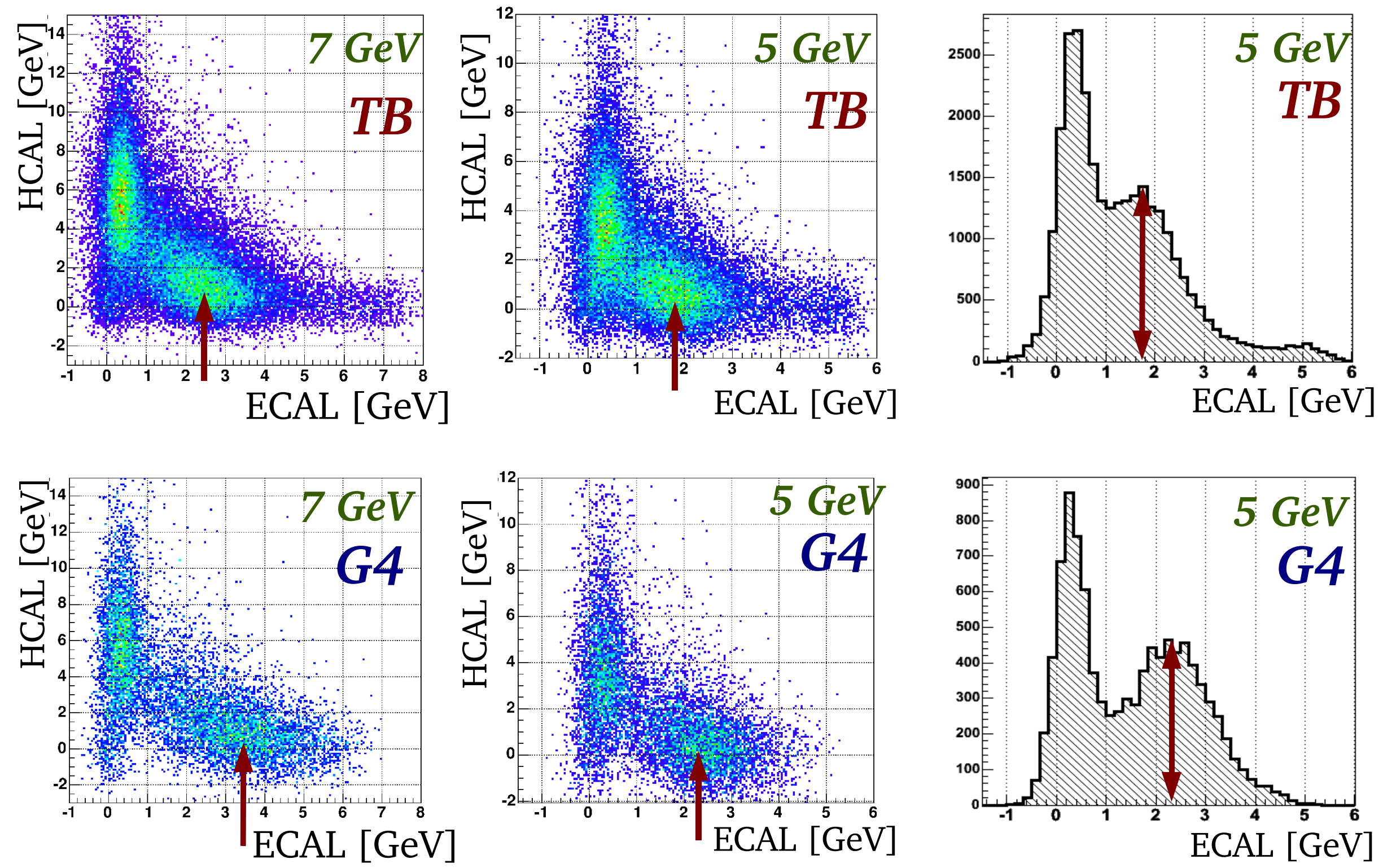

ECAL response is higher in GEANT4 at low energy: geometry or physics or ...? 


\section{Cluster-based response compensation}

Fractional energy resolution for pions.

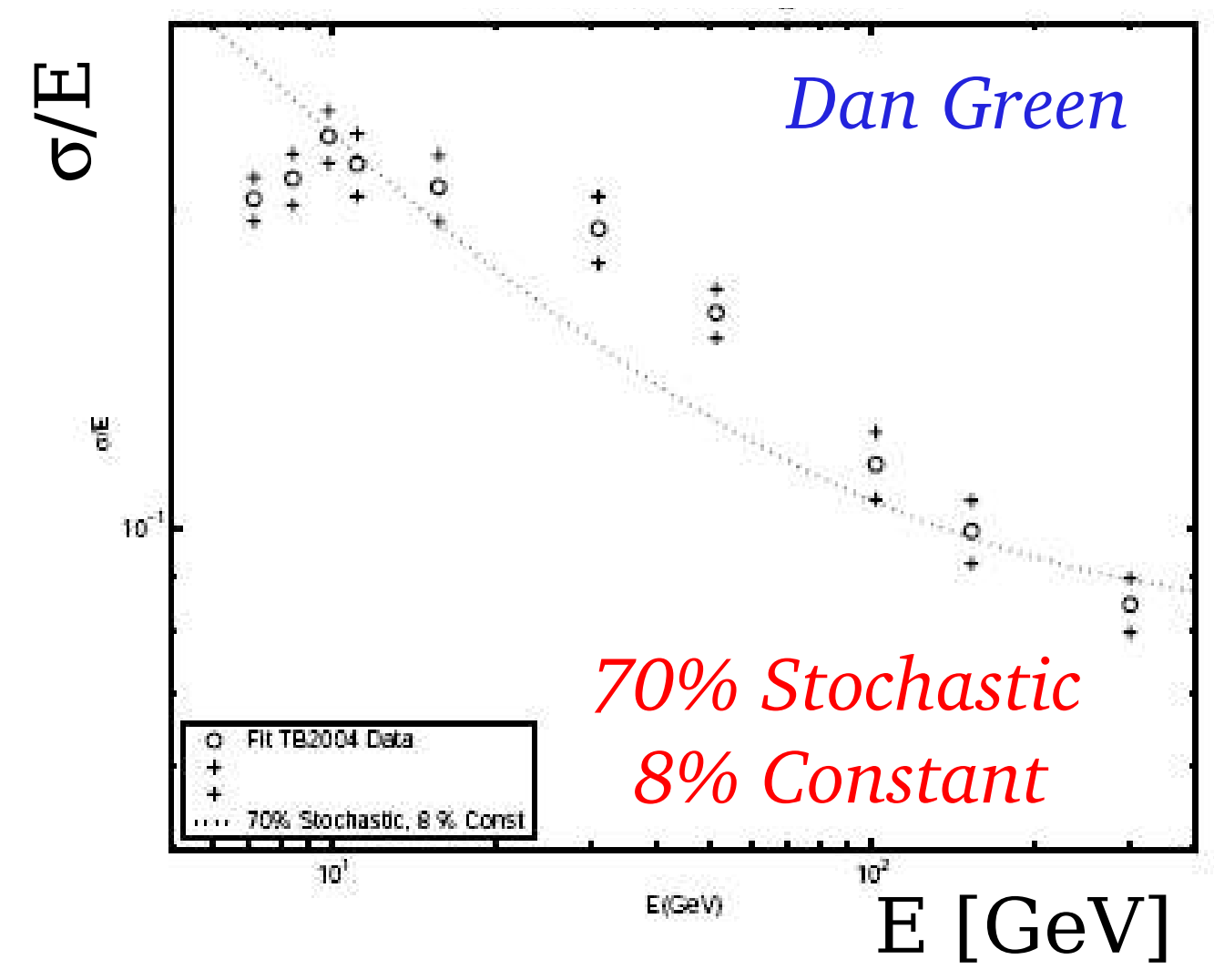

Uses test beam data to fit the intrinsic electron to hadron response $(\mathrm{e} / \mathrm{h})$ and the average neutral fraction $\mathrm{f}_{0}$ of the ECAL and HCAL as a function of the raw total calorimeter energy, $\mathrm{E}+\mathrm{H}$. 


\section{Conclusions and Outlook}

- Calorimeter response for momentum range 5-300 GeV/C was measured with test beam in 2004 .

GEANT4 is in good overall agreement with the data

LHEP shows best agreement

- We observed small discrepancy in the following quantities :

1)Longitudinal shower shape for $150-300 \mathrm{GeV} / \mathrm{c}$ pions, modeled by QGSP physics list

2)Discontinuity in the calorimeter response in 7-10 GeV/C range

3)ECAL response to very low energy pion beam is higher in the GEANT4 simulation .

- We plan to repeat the measurements this summer with ECAL production super-module and improved particle Id. 